Research paper

\title{
Defining universality classes for three different local bifurcations
}

\author{
Edson D. Leonel ${ }^{\mathrm{a}, \mathrm{b}, *}$ \\ a Departamento de Física, UNESP - Universidade Estadual Paulista, Av. 24A, 1515 Bela Vista, 13506-900 Rio Claro, SP, Brazil \\ ${ }^{\mathrm{b}}$ Abdus Salam International Center for Theoretical Physics, Strada Costiera 11, Trieste 34151, Italy
}

\section{A R T I C L E I N F O}

\section{Article history:}

Received 21 July 2015

Revised 31 January 2016

Accepted 8 April 2016

Available online 25 April 2016

\section{Keywords:}

Scaling law

Critical exponents

Local bifurcations

\begin{abstract}
A B S T R A C T
The convergence to the fixed point at a bifurcation and near it is characterized via scaling formalism for three different types of local bifurcations of fixed points in differential equations, namely: (i) saddle-node; (ii) transcritical; and (iii) supercritical pitchfork. At the bifurcation, the convergence is described by a homogeneous function with three critical exponents $\alpha, \beta$ and $z$. A scaling law is derived hence relating the three exponents. Near the bifurcation the evolution towards the fixed point is given by an exponential function whose relaxation time is marked by a power law of the distance of the bifurcation point with an exponent $\delta$. The four exponents $\alpha, \beta, z$ and $\delta$ can be used to defined classes of universality for the local bifurcations of fixed points in differential equations.
\end{abstract}

(c) 2016 Elsevier B.V. All rights reserved.

\section{Introduction}

In nature there are many dynamical systems whose dynamics is controlled by a control parameter(s). As soon it(they) is(are) varied, the behavior of the asymptotic state can be changed in such a way that the qualitative structure of the solution is different after and before the change. The point to where such a qualitative change happens is defined as a bifurcation point.

The bifurcations can be classified in two main types [1,2]: (i) local and (ii) global bifurcations. A local bifurcation happens when a variation of the control parameter leads to a change of stability of a fixed point. Hence the topological modifications in the system can be confirmed by a local investigation near the fixed point. On the other hand, the global bifurcations are observed mainly when invariant structures collide with each other, like invariant manifold and chaotic attractor, leading to a destruction of the chaotic attractor. Such a destruction causes a major change in the global topology of the system which cannot be foreseen by a local analysis of fixed point. Examples of such global bifurcations include the so called crisis event [3-6]. In this paper we concentrate in the local bifurcations. Applications and observations of such bifurcations are wide in the literature and include laser [7-9], population dynamics [10,11], chemical reactions [12,13], electric circuits [14,15], discrete mappings [16,17] and many others [18-22].

Our main goal in this paper is to describe the behavior of the convergence to the fixed point and near it for three different local bifurcations, namely: (i) saddle-node; (ii) transcritical; and (iii) supercritical pitchfork. To do so, we consider two different approaches. The first one is phenomenological and shows that, at the bifurcation point, the convergence to the

\footnotetext{
* Correspondence address: Departamento de Física, UNESP - Universidade Estadual Paulista, Av. 24A, 1515 Bela Vista, $13506-900$ Rio Claro, SP, Brazil. Tel.: +55 1935269174 .

E-mail address: edleonel@rc.unesp.br
} 


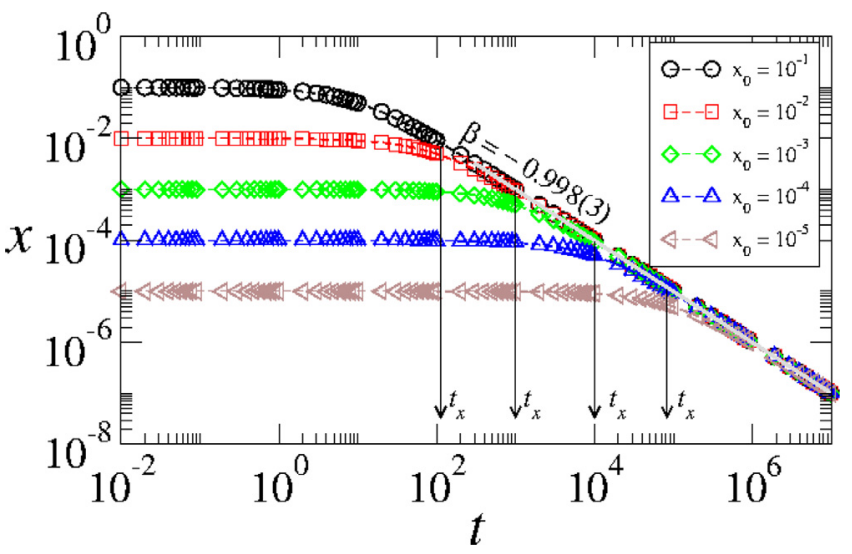

Fig. 1. Plot of $x v$ s. $t$ for Eq. (1) considering different values of $x_{0}$, as show in the figure.

fixed point is described by a homogeneous function with three critical exponents $\alpha, \beta$ and $z$. The exponent $\alpha$ characterizes the dynamics for short time. We shall show that in such a domain, the dynamics is strongly dependent on the initial distance from the fixed point. When the initial condition is given very close to the fixed point, the dynamics stays sticky in a constant plateau for a long time. Hence, the critical exponent $\alpha$ characterizes such a dynamics. For long time, the dynamics changes from this constant regime and enters in a decay towards the equilibrium. The speed of the convergence is given by the exponent $\beta$. The time marking the crossover from one region to the other is characterized by the exponent $z$. A scaling law is obtained relating all the three exponents in a single relation. Therefore the knowledge of only two of them is enough to determine the third. Near the bifurcation, the decay to the fixed point is exponential and the relaxation time is given in terms of a power law from the distance (in terms of the parameter) of the bifurcation with an exponent $\delta$. The knowledge of the four critical exponents define to which universality class the bifurcations belongs to. The present formalism can be used as an alternative to classify the bifurcations mainly, but not only, in experimental systems when the modeling dynamical equations are not available or unknown.

The investigation of such a convergence is made by using two approaches. The first one is phenomenological where a set of three scaling hypotheses are presented together with a scaling function. Numerical simulations are made to obtain an estimation for the critical exponents. The second one comes from the exact solution of the differential equation, where the critical exponents emerge naturally from the dynamics. The second approach validates the first one. As discussed in Refs. [1,2], a generic vector field obtained by the solution of an equation of the type $\dot{x}=f(x, \mu)$, where $x$ is a dynamical variable and $\mu$ is a control parameter and $f$ is a nonlinear function, can be Taylor expanded near the fixed point. As a result, the dynamics can be matched in one of the three main differential equations: (a) $\dot{x}=\mu-x^{2}$ (saddle-node); (b) $\dot{x}=\mu x-x^{2}$ (transcritical); and (c) $\dot{x}=\mu x-x^{3}$ (supercritical pitchfork). This is possible due to a so called normal form theory [1,2].

The paper is organized as follows. In Section 2 we describe the general aspects of the procedure illustrating how the phenomenological investigation is made. The scaling law is derived in Section 2 too. Section 3 is reserved to described the critical exponents in the saddle-node bifurcation while the properties for the transcritical bifurcation are described in Section 4. The critical exponents for the supercritical pitchfork bifurcation is presented in Section 5 . Our discussions and final remarks are given in Section 6.

\section{Convergence to the fixed point}

To start with, let us discuss the convergence to the fixed point for the following differential equation

$$
\dot{x}=\mu-x^{2} \text {. }
$$

There is a saddle-node bifurcation at $\mu=0$. The fixed points are $x_{1,2}^{*}= \pm \sqrt{\mu}$ for $\mu \geq 0$. The fixed point $x_{1}^{*}$ is asymptotically stable for $\mu>0$ while $x_{2}^{*}$ is unstable. It means that any neighboring initial condition of $x_{1}^{*}$ converge to it as time goes. On the other hand, the initial conditions chosen near $x_{2}^{*}$ apart from it as time evolves. The two questions we can pose are: (i) How fast is such a convergence? (ii) Could the convergence depends on the initial $x_{0}$ (the initial distance from the fixed point)? We show that the convergence does indeed depend on the initial $x_{0}$ and that the speed of convergence depends on the equation. Fig. 1 shows a typical behavior of the convergence to the fixed point ( $x v s$. $t$ ) for Eq. (1), at the bifurcation in $\mu=0$, considering different values for $x_{0}$, as shown in the figure.

We can see that, depending on the value of $x_{0}$, the orbit keeps sticky in a constant plateau for a long time. It then experiences a changeover and enters in a regime of decay given by a power law, converging asymptotically to the fixed point. The characteristic time $t_{x}$ that marks the changeover from the constant plateau to the regime of decay depends also on the initial condition $x_{0}$. Let us then describe the convergence to the fixed point in a phenomenological way. Later on the paper we give a mathematical description for it. 
The behavior shown in Fig. 1 allows us to propose the following scaling hypotheses to describe the behavior of $x$ as a function of time $t$ at the bifurcation.

1. For sufficiently short time $t$ the behavior of $x v s$. $t$ is given by

$$
x(t) \propto x_{0}^{\alpha},
$$

for $t \ll t_{x}$, where $x_{0}$ gives indeed the initial distance of the fixed point, $\alpha$ is a critical exponent and $t_{x}$ is a characteristic time that marks the change from the plateau to the regime of decay;

2. For sufficiently long time $t$ we have

$$
x(t) \propto t^{\beta},
$$

for $t \gg t_{x}$, where $\beta$ is a critical exponent that defines the speed of convergence to the stationary state;

3. The characteristic time $t_{x}$ that marks the changeover from the plateau to the regime of decay is given by

$$
t_{x} \propto x_{0}^{z}
$$

where $z$ is a critical exponent that defines the dependence of $t_{x}$ with the initial distance $x_{0}$.

The three scaling hypotheses allow us to describe the fixed point by using a homogeneous generalized function [23] of the type

$$
x\left(x_{0}, t\right)=\ell x\left(\ell^{a} x_{0}, \ell^{b} t\right)
$$

where $\ell$ is a scaling factor, $a$ and $b$ are characteristic exponents.

Because $\ell$ is a scaling factor, we can choose it as

$$
\begin{aligned}
\ell^{a} x_{0} & =1, \\
\ell & =x_{0}^{-1 / a} .
\end{aligned}
$$

Substituting Eq. (6) in (5) we have

$$
x\left(x_{0}, t\right)=x_{0}^{-1 / a} x\left(1, x_{0}^{-b / a} t\right),
$$

where the function $x\left(1, x_{0}^{-b / a} t\right)$ is assumed to be constant for $t \ll t_{x}$. Comparing Eq. (7) with Eq. (2) we conclude $\alpha=-\frac{1}{a}$.

Choosing now

$$
\begin{aligned}
\ell^{b} t & =1, \\
\ell & =t^{-1 / b},
\end{aligned}
$$

and substituting Eq. (8) in Eq. (5) we end up with

$$
x\left(x_{0}, t\right)=t^{-1 / b} x\left(t^{-a / b} x_{0}, 1\right),
$$

where we assume $x\left(t^{-a / b} x_{0}, 1\right)$ is constant for $t \gg t_{x}$. Comparing Eq. (9) with Eq. (3) we conclude $\beta=-\frac{1}{b}$.

Finally we can compare the two expressions obtained for $\ell$ and obtain

$$
t_{x}=x_{0}^{\alpha / \beta}
$$

A comparison of Eq. (10) with Eq. (4) gives

$$
z=\frac{\alpha}{\beta} \text {. }
$$

Eq. (11) defines a scaling law. The knowledge of any two critical exponent allows to obtain the third.

The critical exponents $\alpha, \beta$ and $z$ can be obtained from specific plots. A careful look at Fig. 1 allows us to conclude that for short time, $x(t) \cong x_{0}$, leading to $\alpha=1$. The exponent $\beta$ is obtained from a power law fitting to the decay. As shown in Fig. 1 , we found $\beta=-0.998(3) \cong-1$. Finally the exponent $z$ is obtained from the crossing of the constant plateau with the curve of decay. The behavior of $t_{x} v$ s. $x_{0}$ is shown in Fig. 2. A power law fitting gives us $z=-1.001(1)$.

The knowledge of the critical exponent $\alpha, \beta$ and $z$ allows us to check the existence of an universal behavior for $x(t)$ at the bifurcation point. To do so, the following scaling transformations must be done to the axis of Fig. $1: x(t) \rightarrow x(t) / x_{0}^{\alpha}$ and $t \rightarrow t / x_{0}^{z}$. With these two transformations, all the curves shown in Fig. 1 overlap each other into a single and hence universal curve, as shown in Fig. 3.

Let us now discuss the convergence to the fixed point by considering $\mu>0$. Fig. 4(a) shows the behavior of $x v s$. $t$ for different values of $\mu$. We notice the curves converge to the fixed point at different speeds. The final convergence is shown in the zoom-in of Fig. 4(a). We then suppose that

$$
x(t)-x^{*} \propto e^{-\frac{t}{\tau}},
$$

where $\tau$ is the relaxation time given by

$$
\tau \propto \mu^{\delta}
$$




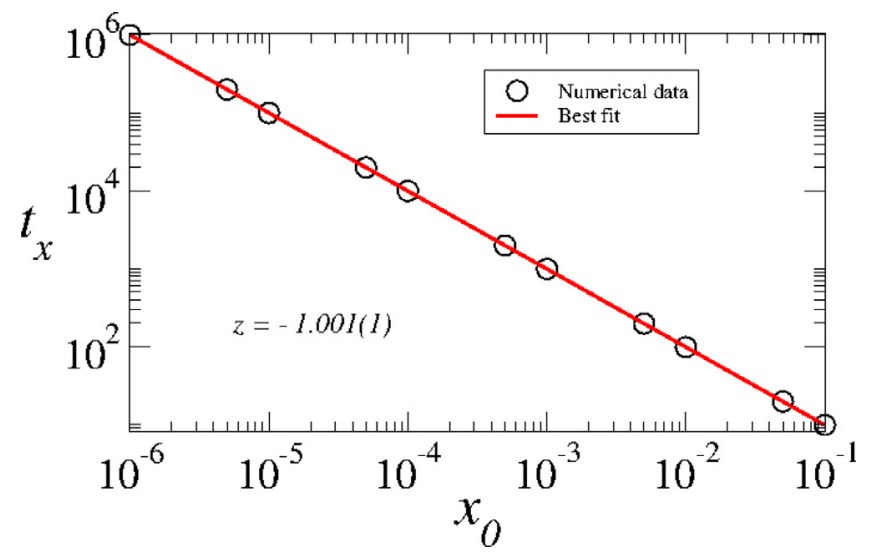

Fig. 2. Plot of $t_{x} v$ s. $x_{0}$ for Eq. (1). A power law fitting furnishes $z=-1.001(1)$.

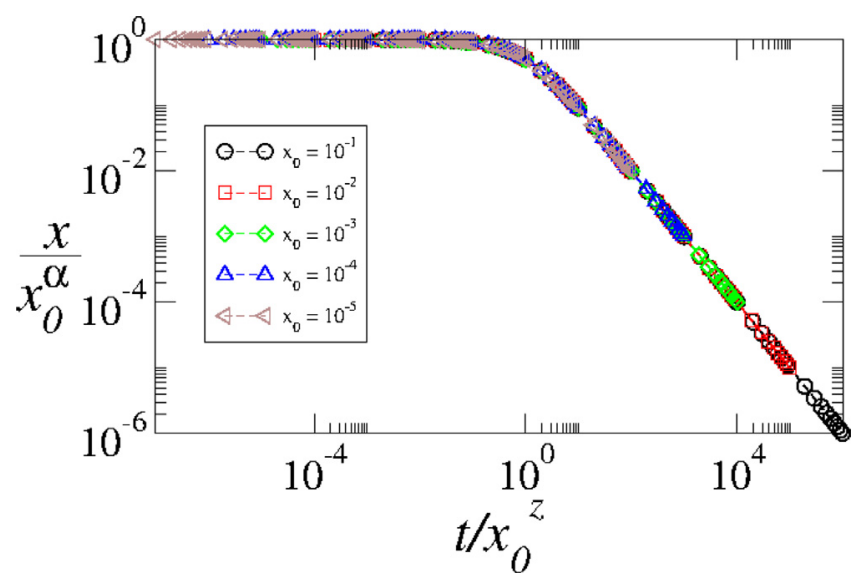

Fig. 3. Overlap of all curves shown in Fig. 1 onto a single and universal plot after the following scaling transformations to the axis: (i) vertical $-x \rightarrow x / x_{0}^{\alpha}$ and; (ii) horizontal $-t \rightarrow t / x_{0}^{z}$.
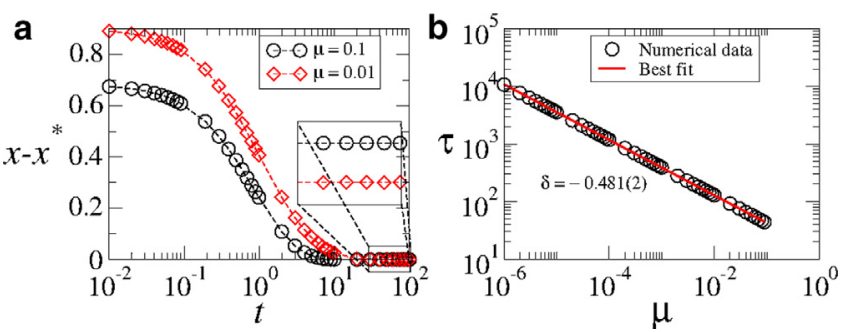

Fig. 4. (a) Plot of $x v$ s. $t$ for Eq. (1) considering $\mu \neq 0$. (b) Plot of $\tau v$ s. $\mu$, hence confirming the existence of a power law with exponent $\delta=-0.481(3) \cong$ -0.5 .

and $\delta$ is a critical exponent. The relaxation time is obtained when the dynamical variable $x$ reaches a distance from the fixed point smaller than $10^{-8}$. The exponent $\delta$ can be obtained by numerical simulations. Fig. 4(b) shows the behavior of the relaxation time $\tau v s$. $\mu$ for Eq. (1). A power law fitting gives us that $\delta=-0.481(3) \cong-0.5$.

The knowledge of the four exponents $\alpha, \beta, z$ and $\delta$ can be used to define universality classes for the bifurcations. We discuss in what follows the procedure to obtain the critical exponents by solving directly the equations for three different types of bifurcations: (i) saddle-node $\left(\dot{x}=\mu-x^{2}\right)$; (ii) transcritical $\left(\dot{x}=\mu x-x^{2}\right)$; and (iii) supercritical pitchfork $(\dot{x}=\mu x-$ $\left.x^{3}\right)$.

\section{Scaling for the saddle-node bifurcation}

Let us discuss in this section how to find the critical exponent $\alpha, \beta, z$ and $\delta$ for the saddle-node bifurcation considering a direct integration of the Eq. (1). Therefore this method is analytic and reinforce our phenomenological approach discussed in 
Section 2. We consider first the case of $\mu=0$. The equation can be written as $\dot{x}=-\chi^{2}$ that leads to the following integration to be solved

$$
\int_{x 0}^{x(t)} \frac{d x}{x^{2}}=-\int_{0}^{t} d t
$$

Doing the integration and grouping properly the terms we obtain

$$
x(t)=\frac{x_{0}}{1+x_{0} t} .
$$

A detailed look at Eq. (15) allows us to obtain the critical exponents. Firstly, for the case of $x_{0} t \ll 1$, we have

$$
x(t) \cong x_{0} .
$$

Comparing Eq. (2) with the first scaling hypothesis we find $\alpha=1$, as we concluded from the Fig. 1.

For $x_{0} t \gg 1$, we have

$$
x(t) \cong t^{-1} \text {. }
$$

A comparison of this result with Eq. (3) from the second scaling hypothesis gives $\beta=-1$, in well agreement with our numerical results.

Finally for $x_{0} t=1$ we have

$$
t_{x} \cong x_{0}^{-1} \text {. }
$$

Comparing this result with Eq. (4) we obtain $z=-1$. Therefore we found analytically the critical exponents $\alpha=1, \beta=-1$ and $z=-1$ for the saddle-node bifurcation. We have to discuss now how to find the critical exponent $\delta$.

To do so, we consider $\mu>0$ in Eq. (1). The equation that must be solved is

$$
\int_{x_{0}}^{x(t)} \frac{d x}{\mu-x^{2}}=\int_{0}^{t} d t
$$

A direct integration of Eq. (19) yields

$$
\left.\operatorname{arctanh}\left[\frac{x}{\sqrt{\mu}}\right]\right|_{x_{0}} ^{x(t)}=\sqrt{\mu} t .
$$

From the identity

$$
\operatorname{arctanh}(x)=\frac{1}{2} \ln \left[\frac{1+x}{1-x}\right],
$$

we can write Eq. (20) as

$$
\left.\ln \left[\frac{1+\frac{x}{\sqrt{\mu}}}{1-\frac{x}{\sqrt{\mu}}}\right]\right|_{x_{0}} ^{x(t)}=2 \sqrt{\mu} t
$$

Grouping the terms properly we end up with

$$
x(t)=\sqrt{\mu}\left[\frac{\left(x_{0}-\sqrt{\mu}\right)+\left(\sqrt{\mu}+x_{0}\right) e^{2 \sqrt{\mu} t}}{\left(\sqrt{\mu}-x_{0}\right)+\left(\sqrt{\mu}+x_{0}\right) e^{2 \sqrt{\mu} t}}\right],
$$

which can be written in a more convenient way as

$$
x(t)=\sqrt{\mu}\left[1+\left(\frac{x_{0}-\sqrt{\mu}}{x 0+\sqrt{\mu}}\right) e^{-2 \sqrt{\mu} t}\right]\left[1-\left(\frac{x_{0}-\sqrt{\mu}}{x_{0}+\sqrt{\mu}}\right) e^{-2 \sqrt{\mu} t}\right]^{-1}
$$

Doing a Taylor expansion to the term inside of the second pair of square brackets and keeping only terms of order $\sqrt{\mu}$ in the equation, we find

$$
x(t)-\sqrt{\mu} \cong\left[\frac{2 \sqrt{\mu} x_{0}}{x_{0}+\sqrt{\mu}}\right] e^{-2 \sqrt{\mu} t} .
$$

The term on the left side of Eq. (25) does indeed give the distance from the fixed point while the right hand gives the exponential decay properly. We see that Eq. (25) is written in the same form as Eq. (12). A comparison, particularly related to the relaxation time given by Eq. (13) allow us to conclude that $\delta=-1 / 2$, which is in remarkably well agreement with the result shown in Fig. 4(b).

We conclude then the critical exponents for the saddle-node bifurcation observed in Eq. (1) are $\alpha=1, \beta=-1, z=-1$ and $\delta=-1 / 2$. 


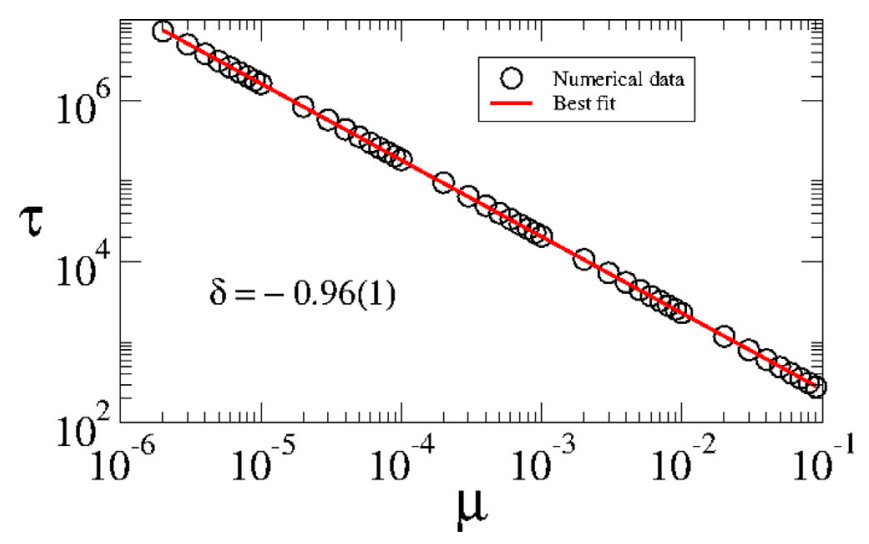

Fig. 5. Plot of $\tau$ vs. $\mu$ for Eq. (26). A power law fitting furnishes $\delta=-0.96(1) \cong-1$.

\section{Scaling for the transcritical bifurcation}

Let us now discuss how to obtain the critical exponents for the transcritical bifurcation. To do so we consider the following equation

$$
\dot{x}=\mu x-x^{2} \text {. }
$$

The fixed points are $x_{1}^{*}=0$, which is asymptotically stable for $\mu<0$ and unstable for $\mu>0$, and $x_{2}^{*}=\mu$ which is unstable for $\mu>0$ and asymptotically stable for $\mu<0$. The change of stability among the two fixed points that happens in $\mu=0$ defines a transcritical bifurcation. For $\mu=0$, Eq. (26) has the same form of Eq. (14). Hence, the dynamics is the same as shown in Fig. 1 leading to the following critical exponents $\alpha=1, \beta=-1$ and $z=-1$. We have now to obtain $\delta$.

For $\mu \neq 0$, the convergence to the fixed point is marked by an exponential function. Fig. 5 shows the behavior of $\tau v$ s. $\mu$. A power law fitting gives $\delta=-0.96(1) \cong-1$.

Let us now discuss the mathematical procedure to obtain the decay for the case of $\mu \neq 0$. The equation to be solved is

$$
\int_{x_{0}}^{x(t)} \frac{d x}{\mu x-x^{2}}=\int_{0}^{t} d t .
$$

After integration and grouping the terms conveniently, we obtain

$$
x(t)=\mu\left[1-\left(1-\frac{\mu}{x_{0}}\right) e^{-\mu t}\right]^{-1} .
$$

Doing a Taylor expansion in Eq. (28) and keeping only linear terms of $\mu$ in the equation, we obtain

$$
x(t)-\mu \cong \mu e^{-\mu t} \text {. }
$$

Eq. (29) is in the same form as Eq. (12). A comparison with Eq. (13) leads to $\delta=-1$, which is in good agreement with our numerical simulation, as shown in Fig. 5. Therefore, the critical exponents for the transcritical bifurcation observed in Eq. (26) are $\alpha=1, \beta=-1, z=-1$ and $\delta=-1$.

\section{Scaling for the supercritical pitchfork bifurcation}

We discuss now the convergence to the fixed point for the supercritical pitchfork bifurcation. The equation we consider is written as

$$
\dot{x}=\mu x-x^{3} \text {. }
$$

The fixed points are $x_{1}^{*}=0$, which is asymptotically stable for $\mu<0$ and unstable for $\mu>0$, and $x_{2,3}^{*}= \pm \sqrt{\mu}$, with $\mu \geq 0$, which are both asymptotically stables. The supercritical pitchfork bifurcation happens at $\mu=0$. Fig. 6(a) shows the behavior of $x v$ s. $t$ at the bifurcation $(\mu=0)$. A power law fitting of the decay furnishes $\beta=-0.4994(1) \cong-0.5$. Since $\alpha=1$ and using the scaling law given by Eq. (11), we find $z=-2$. After a transformation to the axis we see that Fig. 6(a) overlap all curves shown in (a) onto a single and therefore universal plot.

For $\mu \neq 0$, the relaxation to the fixed point is exponential. Fig. 7 shows a plot of $\tau v$ s. $\mu$. A power law fitting gives $\delta=-0.98(1) \cong-1$.

Let us now obtain the critical exponents directly from the solution of Eq. (30). We start first with $\mu=0$, leading to

$$
\int_{x_{0}}^{x(t)} \frac{d x}{x^{3}}=-\int_{0}^{t} d t
$$



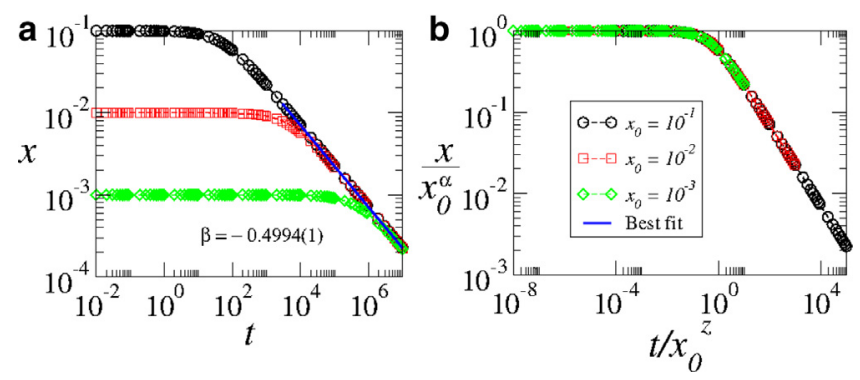

Fig. 6. (a) Plot of $x$ s. $t$ for Eq. (30) considering $\mu=0$ and different values of $x_{0}$, as shown in the figure. (b) Overlap of all curves shown in (a) onto a single and universal plot.

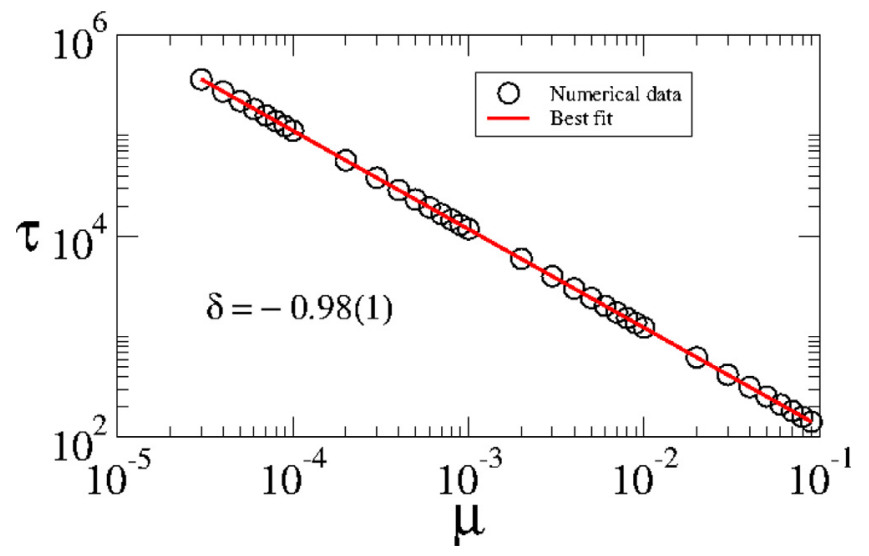

Fig. 7. Plot of $\tau v$ s. $\mu$ for the supercritical pitchfork bifurcation. A power law fitting gives $\delta=-0.98(1) \cong-1$.

After integration and grouping properly the terms we find

$$
x(t)=\frac{x_{0}}{\sqrt{1+2 x_{0}^{2} t}} .
$$

Therefore we have the following conditions:

- For $2 x_{0}^{2} t \ll 1$, we have $x(t) \cong x_{0}$, leading to $\alpha=1$;

- For $2 x_{0}^{2} t \gg 1$, we have $x(t) \cong \frac{1}{\sqrt{2}} t^{-1 / 2}$, leading to $\beta=-1 / 2$;

- Finally for $2 x_{0}^{2} t=1$, we have $t=\frac{1}{2} x_{0}^{-2}$, hence $z=-2$.

The three critical exponents obtained above agree well with our numerical simulations.

The next step is obtain the solution for Eq. (30) considering $\mu \neq 0$. We have to integrate the following equation

$$
\int_{x_{0}}^{x(t)} \frac{d x}{\mu x-x^{3}}=\int_{0}^{t} d t
$$

Integrating and grouping the terms properly leads to

$$
x(t)= \pm \sqrt{\mu}\left[1-\left(\frac{x_{0}^{2}-\mu}{x_{0}^{2}}\right) e^{-2 \mu t}\right]^{-1 / 2} .
$$

Doing Taylor expansion in Eq. (34) and considering only terms of order $\sqrt{\mu}$ in the equation, we obtain

$$
x(t) \mp \sqrt{\mu} \cong \pm \frac{\sqrt{\mu}}{2} e^{-2 \mu t} .
$$

We notice again that Eq. (35) is written in the same form as Eq. (12) leading to $\delta=-1$. We conclude then for the supercritical pitchfork bifurcation observed in Eq. (30), the critical exponents are $\alpha=1, \beta=-1 / 2, z=-2$ and $\delta=-1$.

\section{Discussions and conclusions}

We have studied in this paper the convergence to the fixed point at, and near at, three different local bifurcations of fixed points, namely: saddle-node, transcritical and supercritical pitchfork, all in differential equations. At the bifurcation 
Table 1

Table of critical exponents $\alpha, \beta, z$ and $\delta$ for the three bifurcations discussed in this paper.

\begin{tabular}{llclll}
\hline Equation & Bifurcation & $\alpha$ & $\beta$ & $z$ & $\delta$ \\
\hline$\dot{x}=\mu-x^{2}$ & Saddle-node & 1 & -1 & -1 & $-\frac{1}{2}$ \\
$\dot{x}=\mu x-x^{2}$ & Transcritical & 1 & -1 & -1 & -1 \\
$\dot{x}=\mu x-x^{3}$ & Supercritical pitchfork & 1 & $-\frac{1}{2}$ & -2 & -1 \\
\hline
\end{tabular}

Table 2

Table of critical exponents $\alpha, \beta, z$ and $\delta$ observed for a family of logistic-like map $x_{n+1}=$ $R x_{n}\left(1-x_{n}^{\gamma}\right)$ (see Ref. [24] for more details).

\begin{tabular}{lllll}
\hline Bifurcation & $\alpha$ & $\beta$ & $z$ & $\delta$ \\
Pitchfork & 1 & $-\frac{1}{\gamma}$ & $-\gamma$ & -1 \\
Transcritical & 1 & $-\frac{1}{\gamma}$ & $-\gamma$ & -1 \\
Period doubling & 1 & $-\frac{1}{2}$ & -2 & -1 \\
\hline
\end{tabular}

point, the convergence to the fixed point is given by a homogeneous function with three critical exponents $\alpha, \beta$ and $z$. All of them are related among each other via a scaling law $z=\alpha / \beta$. Near the bifurcation, the convergence to the fixed point is given by an exponential function and the relaxation time is marked by a power law with exponent $\delta$. The four exponents define the class of universality the bifurcation belongs to. Table 1 shows the four exponents for the bifurcations considered in this paper.

As discussed in Refs. [1,2] and due to the representation of the so called normal forms, the three bifurcations presented here are the prototypes of such bifurcations in a widely number of systems described by differential equations. For a generic vector field produced by a differential equation of the type $\dot{x}=f(x, \mu)$, where $x$ is the dynamical variable and $\mu$ is a control parameter, the normal form allows one to Taylor expand the function $f(x, \mu)$ at the lower nonlinearity near the fixed point, which may be fitted in one of the three cases discussed here. We have not considered the subcritical pitchfork bifurcation $\dot{x}=\mu x+x^{3}$ since $x=0$ is unstable at the bifurcation, nor the Hopf bifurcation will be made later on.

Finally we have to mention the approach used here has been applied with success in the investigation of fixed point convergence in a family of logistic-like mappings [24] of the type $x_{n+1}=R x_{n}\left(1-x_{n}^{\gamma}\right)$. The investigation was carried out for different values of $\gamma$. For any even $\gamma, R=1$ gives a transcritical bifurcation and the critical exponents are summarized in Table 2. For any other $\gamma$ (odd, irrational etc.), $R=1$ gives a pitchfork bifurcation, whose critical exponents are also shown in Table 2. The period doubling bifurcation at $R=(2+\gamma) / \gamma$ leads to universal exponents which do not depend on $\gamma$, as they do indeed depend on the pitchfork and transcritical bifurcations. The critical exponents for the pitchfork and transcritical are the same with $\beta$ and $z$ dependent on the nonlinearity of the mapping. In differential equations, the only different exponent from the saddle-node and transcritical bifurcations is $\delta$, while it is the same for the logistic-like map. The period doubling bifurcation in logistic-like map however shows to have the same set of critical exponents as for the supercritical pitchfork observed in the paper. An analytical investigation confirms this finding [25].

In summary, the knowledge of the four critical exponent can be used to defined and characterize classes of universality to bifurcations of fixed points in either mappings and differential equations. The approach can be considered an alternative to define the bifurcation type in experimental systems when no modeling equations are available.

\section{Acknowledgments}

EDL acknowledges support from FAPESP (2012/23688-5), CNPq (303707/2015-1) and FUNDUNESP, Brazilian agencies.

\section{References}

[1] Wiggins S. Introduction to applied nonlinear dynamical systems and chaos. New York: Springer-Verlag; 1990.

[2] Guckenheimer J, Holmes P. Nonlinear oscillations, dynamical systems and bifurcations of vector fields. New York: Springer-Verlag; 1986.

[3] Grebogi C, Ott E, Yorke JA. Phys Rev Lett 1983;48:1507.

[4] Grebogi C, Ott E, Yorke JA. Physica D 1983;7:181.

[5] Leonel ED, McClintock PVE. J Phys A Math Gen 2005;38:L425.

[6] Oliveira DFM, Leonel ED, Robnik M. Phys Lett A 2011;375:3365.

[7] Virte M, Panajotov K, Thienpont H. Nat Photonics 2013;7:60.

[8] Doedel EJ, Pando-L CL. Phys Rev E 2011;84:056207.

[9] Cavalcante HLDS, Leite-Rios JR. Chaos 2008;18:023107.

[10] Bashkirtseva I, Ryashko L. Chaos 2011;21:047514.

[11] Strogatz SH. Physica D 2000;143:1.

[12] Inarrea M, Palacian JF, Pascual AI. J Chem Phys 2011;135:014110.

[13] Bakes D, Schreiberova L, Schreiber I. Chaos 2008;18:015102.

[14] Georgiou IT, Romeo F. Int J Nonlinear Mech 2015;70:153.

[15] Gardine L, Fournier-Prunaret D, Charge P. Chaos 2011;21:023106.

[16] Guckenheimer J. Chaos 2008;18:015108. 
[17] Philominathan P, Santhiah M, Mohamed IR, Murali K, Rajasekar S. Int J Bifurc Chaos 2011;21:1927.

[18] Yang JH, Sanjuán MAF, Liu HG, Cheng G. J Comput Nonlinear Dyn 2015;10:061017.

[19] Guo Y, Luo ACJ. Int J Bifurc Chaos 2012;22:1250268.

[20] Luo ACJ, O’Connor D. Int J Bifurc Chaos 2009;19:2093.

[21] Strogatz SH. Nonlinear dynamics and chaos: with applications to physics, biology, chemistry and engineering. Bolder: Westview Press; 2015.

[22] Lichtenberg AJ, Lieberman MA. Regular and chaotic dynamics. New York: Springer-Verlag; 1992.

[23] Pathria RK. Statistical mechanics. Burlington: Elsevier; 2008

[24] Teixeira RMN, Rando DS, Geraldo FC, Costa-Filho RN, Oliveira JA, Leonel ED. Phys Lett A 2015;379:1246.

[25] Leonel ED, Teixeira RMN, Rando DS, Costa-Filho RN, Oliveira JA. Phys Lett A 2015;379:1796. 